ARTÍCULOS

\title{
Coordinación de estrategias de uso de los recursos de especies leñosas del matorral de Chile: Relaciones entre rasgos funcionales de copa, hoja, tallo, raíz y fenología
}

\author{
Resource-use strategy coordination across woody species of the Chilean matorral: \\ Relationships among functional traits of crown, leaf, stem, root and phenology
}

\author{
Ariel Isaías Ayma-Romay ${ }^{\text {a, b*, Horacio E Bown }}{ }^{\text {c }}$ \\ *Autor de correspondencia: a Universidad de Chile, Programa de Doctorado en Ciencias Silvoagropecuarias y Veterinarias, \\ Campus Sur, Santa Rosa 11315, Santiago, Chile, tel: 56-29785762, ariel.isaias.aymar@gmail.com \\ ${ }^{\mathrm{b}}$ Universidad Católica Boliviana San Pablo, Departamento de Ciencias Exactas e Ingeniería, \\ Centro de Investigación en Ciencias e Ingeniería (CICEI), M. Márquez esq. Plaza Trigo, Cochabamba, \\ Bolivia. tel: 591 - 4 - 4291145, aayma@ucb.edu.bo \\ c Universidad de Chile, Facultad de Ciencias Forestales y Conservación de la Naturaleza, Santiago, Chile, hbown@uchile.cl
}

\section{SUMMARY}

Variation of functional traits among species allows evaluating their life strategies. The whole-plant economic spectrum proposes coordination of the form and function across different plant organs to optimize a resource-use strategy. However, few studies have evaluated the coordination among woody species through functional traits from different plant organs. We evaluated variation and coordination of crown, leaf, stem, root and phenology trait values across woody species of the semi-arid Mediterranean matorral of Chile. We hypothesize that sclerophyllous and malacophyllous woody species vary and coordinate their trait values along an ecological dimension that separates their resource-use strategies from conservative to acquisitive, respectively. We analyzed the variation and correlation of 14 functional traits for 12-16 species. The functional trait values between sclerophyllous and malacophyllous species did not differ across the whole-plant economic spectrum. Traits were coordinated along multiple ecological dimensions. The first dimension was associated with the leaf economic spectrum and plant morphology, yet differed between sclerophyllous and malacophyllous species. Sclerophyllous species combined mostly conservative foliar trait values (longer leaf life span) with acquisitive morphological trait values (larger maximum plant height and longer lateral extension of roots), while malacophyllous species exhibited opposite combinations. The second dimension was associated with variation in phenology and root traits. This dimension separated species with conservative phenological and root trait values (delayed growth onset and deeper rooting depth) from opportunistic species with opposite trait values. The third dimension was coordinated by wood density and the leaf ${ }^{13} \mathrm{C}$ - isotopic composition. This dimension separated the drought-tolerant species with high stem densities and higher leaf water use efficiency from opportunistic water-use species with opposite attributes. In contrast with our hypothesis, in the Chilean matorral the species can combine conservative and acquisitive trait values to use resources and coordinate their resource use strategies across multiple dimensions. This decoupling of plant strategies could occur because different organs and phenology are exposed to different environmental filters.

Key words: functional diversity, Mediterranean, spectrum, leaf economy, root, phenology.

\section{RESUMEN}

La variación de rasgos funcionales entre especies permite evaluar sus estrategias de vida. El espectro de la economía de la planta propone una coordinación de la forma y función de diferentes órganos de la planta para maximizar el uso de recursos. Sin embargo, existen pocos estudios evaluando la coordinación de estrategias de uso de recursos de las especies a través de rasgos funcionales que se encuentran en diferentes órganos de la planta. En este estudio se evalúa la variación y coordinación de valores de rasgos funcionales de copa, hoja, tallo, raíz y fenología relacionados con el uso de los recursos a través de especies leñosas del matorral de Chile. La hipótesis es que las especies leñosas esclerófilas y malacófilas varían y coordinan sus valores de rasgos funcionales a lo largo de una dimensión ecológica que separa sus estrategias de uso de recursos de conservativa a adquisitiva, respectivamente. Las diferencias y correlaciones de 14 rasgos funcionales fueron analizadas a través de 12-16 especies leñosas. Los valores de rasgos funcionales entre especies esclerófilas y malacófilas no difirieron significativamente para ocho de los 15 rasgos funcionales evaluados. La coordinación de valores rasgos funcionales fue a través de múltiples dimensiones ecológicas. En la primera dimensión coordinaron el espectro de la economía de la hoja y la morfología de la planta y fue la única dimensión que separó a las especies esclerófilas de las malacófilas. Las especies esclerófilas combinaron atributos foliares conservativos (mayor longevidad de hoja) y atributos morfológicos adquisitivos (mayor altura máxima de planta y mayor extensión lateral de raíces), mientras las especies malacófilas combinaron atributos opuestos. La segunda dimensión 
separó a las especies que combinaron valores de rasgos fenológicos conservativos (inicio de crecimiento tardío) y una mayor profundidad de raíces y a las especies oportunistas que combinaron atributos opuestos. En la tercera dimensión coordinaron la densidad del tallo y la composición isotópica foliar de ${ }^{13} \mathrm{C}$. Esta dimensión separó a las especies tolerantes al déficit hídrico que combinaron altas densidades de tallo y mayor eficiencia en el uso de agua en la hoja y a las especies oportunistas para el uso del agua que combinaron atributos opuestos. En contraste a nuestra hipótesis, en el matorral de Chile las especies combinan valores de rasgos conservativos y adquisitivos para usar los recursos y coordinan sus estrategias de uso de los recursos a través de múltiples dimensiones. Posiblemente, este desacoplamiento de estrategias ocurre porque diferentes órganos de la planta y su fenología están expuestos a diferentes filtros ambientales.

Palabras clave: estrategia, rasgo funcional, mediterráneo, espectro, raíz, fenología.

\section{INTRODUCCIÓN}

Las plantas adoptan diferentes estrategias de uso de los recursos según las condiciones ambientales en las que habitan. La variación de rasgos funcionales relacionados con el uso de los recursos a través de las especies puede reflejar los compromisos o "trade-offs" que tienen las plantas entre crecimiento y sobrevivencia. Por ejemplo, las especies que habitan ambientes con mayor disponibilidad de recursos exhiben valores de rasgos adquisitivos relacionados con una estrategia de crecimiento rápido y baja inversión en la construcción de estructuras, mientras que las especies que habitan ambientes estresantes muestran valores de rasgos conservativos relacionados con una estrategia de crecimiento lento y alta inversión en la protección de sus órganos (Grime 2001, Reich 2014).

Desde una aproximación de la ecología funcional basada en rasgos, las estrategias de adquisición o conservación de los recursos de las plantas pueden comprenderse evaluando patrones de variación y covariación de rasgos funcionales a través de diferentes especies (Díaz et al. 2004). El espectro de la economía de la hoja (un patrón de variación y covariación de rasgos foliares) muestra en un extremo a las especies con una estrategia de rápida adquisición de carbono y nutrientes con mayor área foliar específica, contenido de nitrógeno, fósforo y tasa de fotosíntesis, y en el otro extremo a las especies con una estrategia de lenta adquisición de esos recursos y mayor inversión en la construcción de estructuras y mecanismos de defensa que combinan valores de rasgos opuestos (Wright et al. 2004). Asimismo, el espectro de la economía de la madera muestra en extremos opuestos a las especies de estrategia adquisitiva con alta conductancia hidráulica y a las especies conservativas con alta resistencia al embolismo y a los daños mecánicos (Chave et al. 2009). Por último, Roumet et al. (2016) mostró un espectro de la economía de las raíces que muestra en un extremo a las especies adquisitivas que combinan mayores tasas de adquisición de agua y nutrientes, mayores tasas de respiración, altas tasas de descomposición, mayor longitud específica de la raíz, y mayor concentración de nitrógeno; en el otro extremo, muestra a las especies conservativas que combinan valores de rasgos opuestos.

En adición, la variación en los rasgos morfológicos de la copa, las raíces y la fenología también reflejaría las es- trategias de uso de los recursos de las especies. Las especies con mayor altura de planta exhiben una estrategia de alta demanda radiativa (estrategia adquisitiva), mientras que las especies de menor altura exhiben una estrategia tolerante a la sombra (estrategia conservativa) (Poorter et al. 2012, de la Riva 2016). Asimismo, en ambientes mediterráneos con marcados cambios estacionales, las especies que tienen un inicio temprano de crecimiento (con respecto a la estación lluviosa) o mayor duración de crecimiento reflejan una estrategia fenológica oportunista que permite capturar los recursos (agua y nutrientes) en los primeros eventos de lluvia o durante largos periodos; mientras que las especies con un inicio tardío de crecimiento y de corta duración de crecimiento muestran una estrategia fenológica conservativa que evita exponer el crecimiento a condiciones ambientales extremas, como bajas temperaturas de invierno y las altas temperaturas de verano (Castro-Díez et al. 2003). Similarmente, las especies de raíces profundas, típicas de ambientes más áridos podrían tener una estrategia conservativa para el uso del agua y de los nutrientes (como mayor densidad de tejidos y menor concentración de nitrógeno), mientras que las especies de raíces superficiales podrían tener una estrategia adquisitiva para el uso del agua (Moreno-Gutiérrez et al. 2012).

Acorde al espectro de la economía de la planta, las especies podrían coordinar diferentes atributos de forma y función a través de diferentes órganos y converger dentro una única estrategia adquisitiva o de lo contrario hacia una estrategia conservativa para adaptarse a su ambiente (Reich 2014). En línea con esta hipótesis, en la Cuenca del Mediterráneo de España las especies leñosas perennes combinaron valores de rasgos que sugieren una coordinada estrategia conservativa a través de diferentes órganos (menor área foliar específica, mayor densidad de tallo y menor longitud específica de la raíz), mientras que las especies leñosas deciduas mostraron una coordinada estrategia adquisitiva para el uso de los recursos con valores de rasgos opuestos (de la Riva et al. 2016). Similarmente, en bosques subtropicales de China las especies leñosas perennes exhibieron coordinadamente una estrategia conservativa a través de rasgos de copa, tallo y hoja (por ejemplo, combinaron menor altura de planta, mayor densidad de madera, mayor contenido foliar de materia seca) para incrementar su tolerancia a la sombra, mientras las especies leñosas deciduas exhibieron una coordinada estrategia adquisitiva combi- 
nando valores de rasgos opuestos para aumentar su habilidad competitiva para capturar la luz (Zhao et al. 2016). Asimismo, especies herbáceas y leñosas con mayor altura de planta podrían coordinar con raíces menos profundas y lateralmente más extensas para capturar mayor cantidad de recursos (Grime 2001). Finalmente, especies que combinan valores de rasgos de hojas y tallos que reflejan una estrategia adquisitiva (menor contenido de materia seca de la hoja y menores densidades de madera) podrían tener un inicio de crecimiento temprano respecto a la temporada de lluvia, lo que reflejaría su estrategia oportunista para capturar el agua (Lima y Rodal 2010).

En contraste, existen estudios que muestran una débil coordinación entre rasgos de hoja, tallo y raíz (Baraloto et al. 2010, Fortunel et al. 2012, Silva et al. 2018, Delpiano et al. 2020) o entre diferentes atributos de hoja y fenología (Castro-Díez et al. 2003, Díaz et al. 2004) que contradicen lo propuesto por el espectro de la economía de la planta. Posiblemente, la escasa coordinación de rasgos funcionales ocurre porque existe un desacople entre funciones para el uso del agua y para el uso del carbono entre o dentro de diferentes órganos. Por ejemplo, la conductancia hidráulica y la seguridad biomecánica en la hoja no siempre están inversamente relacionados (Méndez-Alonzo et al. 2019). Asimismo, diferentes órganos pueden desarrollar estrategias de uso de los recursos independientes en respuesta a diferentes filtros de estrés ambiental a través del espacio o el tiempo (Weemstra et al. 2016). Por ejemplo, los filtros ambientales existentes arriba y abajo del suelo, pueden ser diferentes, lo que evita que los rasgos funcionales de hoja y raíz coordinen hacia una única estrategia de uso de los recursos (Carvajal et al. 2019, Delpiano et al. 2020). Asimismo, los filtros ambientales podrían actuar a través del tiempo afectando de manera diferente a los filtros que actúan a través del espacio.

En bosques de clima mediterráneo el déficit hídrico predomina debido a la fuerte estacionalidad y las precipitaciones poco predecibles (Rundel et al. 2016). Acorde a la hipótesis del espectro de la economía de la planta en este tipo de clima podríamos esperar una fuerte coordinación de rasgos funcionales de uso de los recursos de las especies a través de diferentes órganos de las plantas. De hecho, en ambientes mediterráneos las especies esclerófilas perennes y las malacófilas deciduas tienen una coordinada estrategia conservativa o adquisitiva para el uso de los recursos, respectivamente, al menos a nivel de hoja (Moreno-Gutiérrez et al. 2012, Prieto et al. 2018, Querejeta et al. 2018). Además, esta coordinación de estrategias para el uso de los recursos podría extenderse a través de diferentes órganos de las plantas, al menos en ecosistemas mediterráneos (Canadell y Zedler 1994, Pérez-Ramos et al. 2012, de la Riva et al. 2016). Una estrategia conservativa a través de todos los órganos de las plantas permitiría a las especies esclerófilas perennes mantener reducidas tasas de fotosíntesis y transpiración durante todo el año, mientras una estrategia adquisitiva permitiría a las especies malacófilas deciduas tener altas tasas de fotosíntesis y transpiración durante un breve periodo de mayor disponibilidad de recursos para crecer (Mooney y Dunn 1970). Sin embargo, ese patrón de coordinación podría no ser una la regla para las regiones mediterráneas, ya que en los chaparrales de California las especies leñosas exhiben una débil coordinación entre rasgos funcionales relacionados con el uso del carbono (a nivel de hoja) y el uso del agua (a nivel de tallo) (Ackerly 2004). Esos estudios sugieren que existe evidencia contradictoria sobre los patrones de coordinación de estrategias de uso de los recursos de especies leñosas a través de diferentes órganos de la planta y la fenología. Por tanto, es importante evaluar patrones de coordinación de rasgos funcionales a través de diferentes órganos de la planta para comprender de mejor forma las estrategias de uso de recursos de las especies.

Este estudio tiene el objetivo de evaluar la variación y coordinación de rasgos de copa, hoja, tallo, raíz y fenología a través de especies leñosas del matorral de Chile para comprender sus estrategias para usar los recursos. Por lo que se conoce, este es el primer estudio que reporta la coordinación de diferentes rasgos funcionales de las plantas perteneciendo a diferentes órganos de las plantas y además incluyendo rasgos de difícil medición como la morfología de raíces y la fenología en ambientes mediterráneos. Se formula la siguiente pregunta ¿Cómo coordinan rasgos morfológicos de copa, hoja, tallo, raíz y fenología para usar los recursos en especies leñosas esclerófilas y malacófilas del matorral de Chile? La hipótesis del estudio es que las especies esclerófilas perennes y malacófilas deciduas tienen contrastantes atributos de copa, hoja, tallo, raíz y fenología, los cuales reflejan sus diferentes estrategias para usar los recursos. Si esto ocurre los atributos de las especies varían y coordinan a través de una dimensión que separa en un extremo a las especies esclerófilas perennes con rasgos conservativos para usar los recursos y en el otro extremo a las especies malacófilas deciduas con rasgos adquisitivos.

\section{MÉTODOS}

Área de estudio. El estudio se realizó en la Reserva Nacional Roblería de Los Cobres de Loncha (en adelante reserva de Loncha) (34 7' $36^{\prime \prime} \mathrm{S}, 71^{\circ} 11^{\prime} 18^{\prime \prime}$ OE) y en la localidad colindante de Los Bronces, Chile ( $34^{\circ} 11^{\prime} 13^{\prime \prime}$ S, 71 1' 7" $^{\circ}$ $\mathrm{OE}$ ). El área corresponde a los cordones orientales de la Cordillera de la Costa. El clima es de tipo mediterráneo semiárido con una época lluviosa fría y una época seca cálida de seis meses. Acorde a la estación climatológica de Rancagua, la localidad más próxima al área de estudio, la reserva de Loncha tiene una precipitación promedio anual de $474 \mathrm{~mm}$ y una temperatura anual de $14,7^{\circ} \mathrm{C}$. La topografía es montañosa, con pendientes moderadas a empinadas. Los suelos están formados por materiales coluviales. La vegetación del matorral dentro y alrededor de la reserva se distribuye de 500 a 900 m s.n.m. Los matorrales son 
formaciones que varían en composición de bosques esclerófilos perennes dominados por peumo (Cryptocarya alba (Molina) Looser) y litre (Lithraea caustica Hook et Arn) a bosques espinosos malacófilos deciduos dominados por espino (Acacia caven (Molina) Molina) y trevo (Retanilla trinervia (Guillies et Hook) Hook et Arn). En general, los matorrales esclerófilos perennes y los matorrales malacófilos deciduos se distribuyen a través de una gradiente de mayor a menor disponibilidad de agua (Bown et al. 2014), y de menor a mayor disponibilidad de luz (Armesto y Martinez 1978), respectivamente.

Selección de especies. Las especies seleccionadas fueron las de mayor abundancia relativa en la reserva de Loncha. Estas especies cubrieron un amplio rango de especies arbóreas y arbustivas, y además diferentes morfologías de hoja (esclerófila y malacófila) y hábitos de vida (perennes, deciduas y semi-deciduas) en línea con previos estudios (Montenegro et al. 1989) (cuadro 1).

Selección de rasgos funcionales. Catorce rasgos funcionales de la copa, tallo, hoja, raíz y fenología fueron seleccionados y relacionados con las estrategias de uso de los recursos. Estos rasgos se relacionaron con funciones de la planta relevantes para el uso de los recursos (agua, nutrientes, luz, carbono) (Pérez-Harguindeguy et al. 2013) (cuadro 2).
Medición de rasgos. Los rasgos funcionales fueron obtenidos de 11 a 16 especies dependiendo del rasgo. La mayor parte de los rasgos fueron obtenidos por mediciones directas y una menor parte complementada por literatura. El número de plantas medidas por especie para obtener el valor promedio de cada especie fue de 2 a 56 plantas dependiendo del rasgo (apéndice 1 y 2).

La altura máxima de la planta $(\mathrm{H})$ fue medida como la distancia entre el suelo y la altura máxima de la copa de la planta. El diámetro de copa (DC) fue medido como el promedio de la distancia horizontal entre los bordes de la copa medida en dos direcciones opuestas. La $\mathrm{H}$ y el DC se obtuvieron de 6 a 56 plantas por especie. Las plantas se midieron a través de 15 parcelas de muestreo de $25 \times 25$ $m$ que se encuentran en la reserva de Loncha. La medición de $\mathrm{H}$ fue realizada con un hipsómetro (SUUNTO PM-5, Finlandia) y el DC con una cinta métrica.

La profundidad de raíz (PR) fue medida como la distancia vertical entre el cuello de la raíz de la planta hasta la punta de la raíz más profunda. La extensión lateral de enraizamiento (ELR) fue la distancia horizontal de la raíz desde el centro de la planta hasta el extremo más lejano dispuesta horizontalmente en los primeros $50 \mathrm{~cm}$ de profundidad del suelo. Ambos rasgos de raíz fueron medidos en 2 a 17 plantas dependiendo de la especie. La medición fue realizada directamente en raíces completas extraídas en un

Cuadro 1. Descripción de las especies seleccionadas para evaluar rasgos funcionales del matorral de Chile de acuerdo al hábito de hoja: esclerófila perenne (ep), malacófila decidua (md), malacófila semi-decidua (msd) y malacófila perenne (mp) y forma de vida.

Description of woody species selected to assess functional traits of the Chilean matorral. Leaf habit: evergreen sclerophyllous (ep), deciduous malacophyllous (md), semi-deciduous malacophyllous (msd), malacophyllous evergreen (mp).

\begin{tabular}{|c|c|c|c|}
\hline Especie & Familia & Forma de vida & Habito de hoja \\
\hline Peumus boldus Molina & Monimiaceae & Árbol & ep \\
\hline Cryptocarya alba (Molina) Looser & Lauraceae & Árbol & ep \\
\hline Kageneckia oblonga Ruiz et Pav. & Rosaceae & Árbol & ep \\
\hline Persea lingue (Miers ex Bertero) Nees & Lauraceae & Árbol & ep \\
\hline Lithraea caustica Hook et Arn & Anacardaceae & Árbol & ep \\
\hline Luma apiculata (DC.) Burret & Myrtaceae & Árbol pequeño & ep \\
\hline Quillaja saponaria Molina & Quillajaceae & Árbol & ep \\
\hline Maytenus boaria Molina & Celastraceae & Árbol & ep \\
\hline Retanilla trinervia (Guillies et Hook.) Hook et Arn & Rhamnaceae & Arbusto & md \\
\hline Retanilla ephedra (Vent.) Brongn. & Rhamnaceae & Arbusto & $\mathrm{md}$ \\
\hline Acacia caven (Molina) Molina & Fabaceae & Árbol & $\mathrm{md}$ \\
\hline Trevoa quinquenervia Guillies et Hook & Rhamnaceae & Árbol pequeño & md \\
\hline Colliguaja odorifera Molina & Euphorbiaceae & Arbusto & msd \\
\hline Sophora macrocarpa $\mathrm{Sm}$ & Caesalpinoideae & Árbol pequeño & msd \\
\hline Baccharis linearis (Ruiz et Pav.) Pers. & Asteraceae & Arbusto & $\mathrm{mp}$ \\
\hline Escallonia pulverulenta (Ruiz et Pav.) Pers. & Escalloniaceae & Árbol pequeño & $\mathrm{mp}$ \\
\hline
\end{tabular}


Cuadro 2. Rasgos funcionales seleccionados para evaluar las estrategias ecológicas de uso de carbono, agua y nutrientes de especies leñosas del matorral de Chile. $\mathrm{Ab}=$ Abreviatura del rasgo.

Functional traits selected to assess ecological strategies to use resources (carbon, water, nutrients) of woody species of the Chilean matorral. $\mathrm{Ab}=$ Abbreviation of functional traits.

\begin{tabular}{|c|c|c|c|c|}
\hline Rasgo planta & $\mathrm{Ab}$ & Unidad & Función & Autores \\
\hline Altura máxima de la planta & $\mathrm{H}$ & $\mathrm{m}$ & $\begin{array}{l}\text { Intercepción de luz, habilidad competitiva, } \\
\text { limitación hidráulica }\end{array}$ & $\begin{array}{l}\text { Pérez-Harguindeguy et al. } \\
\text { (2013) }\end{array}$ \\
\hline Diámetro de copa & $\mathrm{DC}$ & $\mathrm{m}$ & Intercepción de luz & Poorter et al. (2012) \\
\hline Profundidad de raíz & PR & $\mathrm{m}$ & $\begin{array}{l}\text { Adquisición de agua, posición vertical } \\
\text { subterránea }\end{array}$ & Canadell y Zedler (1994) \\
\hline Extensión lateral de la raíz & ELR & $\mathrm{m}$ & Adquisición de nutrientes y agua & Canadell y Zedler (1994) \\
\hline Área foliar & $\mathrm{AF}$ & $\mathrm{cm}^{2}$ & Intercepción solar, transpiración & Wright et al. (2004) \\
\hline Área foliar específica & AFE & $\mathrm{cm}^{2} / \mathrm{g}$ & Tasa fotosintética, tasa de crecimiento & Wright et al. (2004) \\
\hline Contenido foliar de materia seca & CFMS & $\mathrm{g} / \mathrm{g}$ & $\begin{array}{l}\text { Longevidad de la hoja, densidad de tejido, } \\
\text { soporte estructural de la hoja }\end{array}$ & Wright et al. (2004) \\
\hline Longevidad foliar & LF & meses & Adquisición/conservación de nutrientes & Wright et al. (2004) \\
\hline Contenido foliar de nitrógeno & CFN & $\%$ & Capacidad fotosintética de la hoja & Wright et al. (2004) \\
\hline Composición isotópica foliar ${ }^{13} \mathrm{C}$ & $\delta{ }^{13} \mathrm{C}$ & $\%$ & Eficiencia intrínseca de uso de agua & Moreno-Gutiérrez et al. (2012) \\
\hline Densidad específica del tallo & DT & $\mathrm{g} / \mathrm{cm}^{3}$ & Capacidad hidráulica, tasa de crecimiento & Chave et al. (2009) \\
\hline $\begin{array}{l}\text { Tiempo de inicio de crecimiento } \\
\text { de la rama* }\end{array}$ & TICV & días & $\begin{array}{l}\text { Inicio del periodo de crecimiento de la } \\
\text { planta }\end{array}$ & $\begin{array}{l}\text { Montenegro et al. (1979) y } \\
\text { Chuine (2010), }\end{array}$ \\
\hline $\begin{array}{l}\text { Duración del crecimiento } \\
\text { vegetativo de ramas }\end{array}$ & $\mathrm{DCV}$ & días & Periodo de crecimiento de la planta & Castro-Díez et al. (2003) \\
\hline $\begin{array}{l}\text { Tasa de crecimiento relativa de } \\
\text { la rama }\end{array}$ & TCR & $\mathrm{cm} /$ día & Tasa de crecimiento & Grime (2001) \\
\hline
\end{tabular}

(*) Número de días transcurridos desde el 1 de julio de 2017.

predio privado de la localidad de Los Bronces y en raíces de plantas ubicadas en orillas de cortes de camino próximos a la reserva de Loncha. La información fue complementada con estudios realizados en Chile central (apéndice 1 y 2).

La densidad específica del tallo (DT) fue el peso seco del tallo dividido entre el volumen verde. Las muestras de tallo (secciones de 5-8 $\mathrm{cm}$ de diámetro y $10 \mathrm{~cm}$ de largo) fueron recolectadas en $6-21$ plantas por especie en la reserva de Loncha y alrededores. Estas muestras fueron almacenadas en bolsas plásticas por 48 horas y luego remojadas por otras 48 horas. El volumen verde de la sección de tallo con corteza fue obtenido por el método de desplazamiento y el peso seco se obtuvo luego de secarlas por 72 horas a $70^{\circ} \mathrm{C}$ en un horno de aire forzado (PérezHarguindeguy et al. 2013).

$\mathrm{El}$ área foliar (AF), área foliar específica (AFE) y el contenido foliar de materia seca (CFMS) fueron obtenidos en 6 - 20 plantas por especie. De cada individuo se colectaron 10 hojas expuestas al norte totalmente desarrolladas ( $\sin$ peciolo o peciolulos en caso de hojas compuestas) y de tres estratos de la copa (inferior, medio, superior). El área foliar se obtuvo de hojas frescas con fotografías analizadas con ImageJ (version IJ1.46r, National Institutes of Health, Bethesda, MD, USA). El AFE se obtuvo dividien- do el área foliar con el peso seco de cada hoja o conjunto de foliolos para el caso de hojas compuestas. El peso seco de las hojas se obtuvo luego de secarlas a $70{ }^{\circ} \mathrm{C}$ por 72 horas (Pérez-Harguindeguy et al. 2013). El CFMS se obtuvo dividiendo el peso seco de las hojas o foliolos por su peso húmedo (previa hidratación por 48 horas en agua destilada). El contenido foliar de nitrógeno (CFN) y la longevidad foliar (LF) fueron obtenidos de estudios previos publicados y no publicados (apéndice 1 y 2 ).

La composición isotópica foliar de ${ }^{13} \mathrm{C}\left(\delta{ }^{13} \mathrm{C} \%\right)$ fue medida como un rasgo relacionado positivamente con la eficiencia intrínseca de uso del agua (tasa de fotosíntesis/ conductancia estomática) (apéndice 3). La $\delta{ }^{13} \mathrm{C}$ fue obtenida por espectrometría de masa. Las muestras de hoja fueron colectadas de árboles maduros en primavera de 2014 y de 2016 en la reserva de Loncha. En cada temporada se colectaron hojas de 3 a 30 plantas por especie (10 hojas por planta). Las muestras del año 2014 se analizaron en el laboratorio de isotopos estables de la University of Idaho y las muestras del 2016 en el Laboratorio de Suelo-Agua-Planta (SAP) de la Facultad de Agronomía de la Universidad de Chile bajo similares procedimientos. La $\delta$ ${ }^{13} \mathrm{C}$ para cada especie se obtuvo promediando los valores obtenidos en los dos años de muestreo. 
Los rasgos fenológicos fueron el tiempo de inicio de crecimiento vegetativo (TICV), la tasa de crecimiento ( $\mathrm{cm} /$ día) (TCR) y la duración del crecimiento (DCV). Estos rasgos fueron obtenidos en base al crecimiento longitudinal de ramas de las plantas desde el primero de julio de 2017 (fecha de inicio para el seguimiento fenológico) al 30 de marzo de 2018 (fecha final del crecimiento en todas las especies). De cinco a diez plantas por especie fueron marcadas para el seguimiento fenológico. En cada planta se seleccionaron tres ramas de 5 a $10 \mathrm{~cm}$ de longitud preferentemente con exposición noroeste, en las cuales se evaluó el crecimiento longitudinal cada 15-30 días (Montenegro et al. 1979). La TICV expresada en días transcurridos desde el 1 de Julio de 2017 fue calculada como el promedio entre la primera ocurrencia de crecimiento y la fecha anterior de medición sin crecimiento. La DCV se calculó como el número de días transcurridos desde la fecha de inicio de crecimiento (TICV) hasta la fecha de finalización de crecimiento, la cual fue el promedio entre la fecha donde dejo de observarse crecimiento y una fecha anterior donde si hubo crecimiento. La TCR fue obtenida dividiendo el crecimiento acumulado en longitud de cada rama por la duración del crecimiento en días.

Análisis de datos. Las diferencias de los valores de 14 rasgos funcionales entre especies esclerófilas y malacófilas fueron evaluadas con un análisis de varianza de una vía. La normalidad de los datos de cada rasgo fue verificada a través de una prueba de Shapiro-Wilk. Cuando los datos no cumplieron con el supuesto de normalidad fueron transformados mediante logaritmo natural. El patrón de variación y co-variación entre los rasgos de copa, hoja, tallo, raíces y fenología a través de las especies se analizó con un análisis de componentes principales (ACP). El ACP utilizó una matriz de 12 especies y 14 rasgos funcionales. En esta matriz no se incluyeron los rasgos de las 16 especies con el objetivo de reducir número de datos faltantes (principalmente del rasgo de la profundidad de raíces). Aun así, esta matriz presentó cuatro datos faltantes de profundidad de raíces y contenido de nitrógeno foliar los cuales fueron imputados con el método de Random forest. El ACP fue realizado con la matriz de correlaciones y variables escaladas. Para confirmar la fortaleza de correlación entre los rasgos y los ejes de los componentes principales se realizaron pruebas de correlación de Pearson. Al final, el grado de segregación de especies malacófilas y esclerófilas a lo largo de los ejes del ACP fue evaluada con un análisis de varianza utilizando los "scores" de las especies perteneciendo a diferentes grupos funcionales. El programa $\mathrm{R}$ Project versión 3.6.3 (R Core Team, 2020) fue utilizado para el análisis estadístico. El ACP y el análisis de varianza fueron realizados con la función "prcomp" y "anova" del paquete "stats" propio de R, respectivamente. La imputación de datos faltantes en la matriz de especies y rasgos para realizar el ACP fue realizada con el paquete "mice" (van Buuren y Groothuis-Oudshoorn 2011).

\section{RESULTADOS}

En este estudio evaluamos la variación y coordinación de múltiples rasgos de especies leñosas esclerófilas y malacófilas del Mediterráneo de Chile para comprender los patrones de coordinación de sus estrategias para usar los recursos. Los valores de rasgos entre especies esclerófilas y malacófilas no difirieron completamente a través de diferentes órganos de la planta y coordinaron a través de múltiples dimensiones de uso de los recursos. La coordinación de rasgos funcionales que diferencian las estrategias de uso de los recursos a las especies esclerófilas y malacófilas a través de una dimensión no es una regla general en estos ecosistemas mediterráneos.

Las diferencias de valores de rasgos funcionales entre especies esclerófilas y malacófilas. Las especies esclerófilas y las malacófilas difirieron parcialmente a través de diferentes rasgos funcionales. Por un lado, las especies esclerófilas comparadas con las malacófilas mostraron menor área foliar específica, mayor longevidad foliar, menor contenido de nitrógeno, mayor contenido de materia seca, mayor extensión lateral de raíces y altura máxima de la planta, con diferencias estadísticamente significativas. Sin embargo, las especies esclerófilas y malacófilas no tuvieron diferencias estadísticamente significativas en cuanto a su área foliar, composición isotópica foliar de ${ }^{13} \mathrm{C}$, profundidad de raíces, diámetro de copa, densidad del tallo y rasgos fenológicos (cuadro 3).

Variación y coordinación entre rasgos de copa, hoja, tallo, raíz y fenología. El análisis de componentes principales (ACP) mostró que existen tres dimensiones de variación de rasgos funcionales independientes que explican el $77 \%$ de la variabilidad (cuadro 4, figura 1A). El primer componente principal explicó el $43 \%$ de la variabilidad y representó al espectro de la economía de la hoja acoplado al espectro de la morfología de la planta. Por un lado, esta dimensión separó a las especies esclerófilas que combinan valores de rasgos foliares conservativos para la adquisición del carbono (mayor contenido foliar de materia seca y longevidad foliar) y valores de rasgos morfológicos de la planta relacionados con una mayor habilidad competitiva para capturar luz y agua (mayor altura de planta y mayor extensión lateral de raíces). Por otro lado, esta dimensión separó a las especies malacófilas que combinaron valores de rasgos.

El segundo componente principal explicó el $22 \%$ de la variabilidad y mostró una dimensión de rasgos fenológicos que coordinan con la profundidad de raíces y el diámetro de copa. Por un lado, separó a las especies de estrategia oportunista para usar los recursos, las cuales combinaron un inicio de crecimiento temprano, mayor duración de crecimiento, menor tasa de crecimiento de la rama, menor profundidad de raíces y menor diámetro de copa. Por otro lado, separó a las especies de estrategia conservativa, las cuales combinaron un inicio de crecimiento tardío, menor dura- 
Cuadro 3. Análisis de varianza mostrando las diferencias de 14 rasgos funcionales entre especies esclerófilas y malacófilas del matorral de Chile. $\mathrm{n}=$ número de especies, D.E. $=$ Desviación estándar. $P=$ probabilidad de significancia.

Analysis of variance showing differences between sclerophyllous and malacophyllous woody species through 14 functional traits in the Chilean matorral. $\mathrm{n}=$ number of species, D.E. $=$ Standard deviation. $P=$ Probability of significance.

\begin{tabular}{|c|c|c|c|c|c|c|c|}
\hline \multirow{2}{*}{ Rasgo } & \multicolumn{3}{|c|}{ Esclerófilas } & \multicolumn{3}{|c|}{ Malacófilas } & \multirow{2}{*}{$P$} \\
\hline & $\mathrm{n}$ & Media & D.E. & $\mathrm{n}$ & Media & D.E & \\
\hline Altura máxima de la planta (m) & 8 & 7,6 & 2,8 & 8 & 2,66 & 1,33 & $<0,001$ \\
\hline Diámetro de copa (m) & 8 & 4,0 & 1,04 & 8 & 2,66 & 1,62 & 0,08 \\
\hline Densidad del tallo $\left(\mathrm{g} \mathrm{cm}^{3}\right)$ & 8 & 0,62 & 0,07 & 8 & 0,67 & 0,07 & 0,21 \\
\hline Profundidad de raíz (m) & 5 & 3,1 & 1,93 & 6 & 1,82 & 1,69 & 0,27 \\
\hline Extensión lateral de raíz (m) & 7 & 2,8 & 0,86 & 7 & 1,08 & 0,35 & $<0,001$ \\
\hline Área foliar $\left(\mathrm{cm}^{2}\right)^{+}$ & 8 & 11,5 & 9 & 8 & 7,0 & 11 & 0,08 \\
\hline Área foliar específica $\left(\mathrm{cm}^{2} \mathrm{~g}^{-1}\right)^{+}$ & 8 & 81,0 & 29 & 8 & 134,9 & 47 & 0,013 \\
\hline Contenido de materia seca foliar $\left(\mathrm{g} \cdot \mathrm{g}^{-1}\right)$ & 8 & 0,40 & 0,05 & 8 & 0,30 & 0,05 & $<0,001$ \\
\hline Contenido de nitrógeno foliar ${ }^{+}$ & 8 & 1,25 & 0,34 & 6 & 2,08 & 0,91 & $\mathbf{0 , 0 2 7}$ \\
\hline Composición isotópica foliar ${ }^{13} \mathrm{C}(\% \mathrm{c})$ & 8 & $-28,2$ & 1,53 & 8 & $-28,3$ & 1,31 & 0,79 \\
\hline Longevidad foliar (meses) $^{+}$ & 8 & 25 & 15 & 8 & 10,4 & 5 & 0,018 \\
\hline $\begin{array}{l}\text { Tiempo de inicio de crecimiento vegetativo } \\
\text { (número de días desde el } 1 / 7 / 2017 \text { ) }\end{array}$ & 8 & 88 & 19 & 8 & 68 & 38 & 0,22 \\
\hline Duración de crecimiento vegetativo (días) & 8 & 80,7 & 8,7 & 8 & 97,8 & 34,9 & 0,19 \\
\hline Tasa de crecimiento relativo $\left(\mathrm{cm} \mathrm{día}{ }^{-1}\right)^{+}$ & 8 & 0,025 & 0,004 & 6 & 0,028 & 0,013 & 0,75 \\
\hline
\end{tabular}

${ }^{(+)}$Las variables fueron transformadas a escala de logaritmos naturales para realizar las pruebas de Anova.

Valores de $P$ en negrita indican que son significativos a un nivel de significancia de 0,05 .

Cuadro 4. Análisis de componentes principales (ACP) elaborado con una matriz de correlación de 12 especies $\times 14$ rasgos funcionales. Principal component analysis (PCA) prepared with a correlation matrix of 12 species $\times 12$ functional.

\begin{tabular}{|c|c|c|c|c|c|c|}
\hline Variable & CP 1 & $r$ & $\mathrm{CP} 2$ & $r$ & $\mathrm{CP} 3$ & $r$ \\
\hline Proporción acumulada & $43 \%$ & & $65 \%$ & & $77 \%$ & \\
\hline Proporción de varianza explicada & $43 \%$ & & $22 \%$ & & $12 \%$ & \\
\hline Altura máxima de la planta $(\mathrm{H})$ & $-0,29$ & $-0,72 * *$ & $-0,15$ & $-0,27$ & 0,43 & 0,54 \\
\hline Diámetro de copa (DC) & $-0,14$ & $-0,34$ & $-0,39$ & $-0,68 * *$ & 0,22 & 0,29 \\
\hline Densidad del tallo (DT) & 0,26 & $0,63^{*}$ & $-0,12$ & $-0,22$ & $-0,43$ & $-0,55^{*}$ \\
\hline Profundidad de raíz (PR) & $-0,16$ & $-0,37$ & $-0,32$ & $-0,62 *$ & $-0,24$ & $-0,44$ \\
\hline Extensión lateral de raíz (ELR) & $-0,34$ & $-0,83 * * *$ & $-0,14$ & $-0,25$ & 0,03 & 0,03 \\
\hline Área foliar $(\mathrm{AF})^{+}$ & $-0,36$ & $-0,89 * * *$ & 0,04 & 0,07 & 0,09 & 0,12 \\
\hline Área foliar específica $(\mathrm{AFE})^{+}$ & 0,34 & $0,84 * * *$ & $-0,11$ & $-0,20$ & 0,17 & 0,22 \\
\hline Contenido de materia seca foliar (CMSF) & $-0,35$ & $-0,87 * * *$ & 0,01 & 0,02 & $-0,11$ & $-0,14$ \\
\hline Contenido de nitrógeno foliar $(\mathrm{CNF})^{+}$ & 0,35 & $0,81 * *$ & 0,005 & $-0,04$ & 0,17 & 0,30 \\
\hline Composición isotópica foliar ${ }^{13} \mathrm{C}\left(\delta^{13} \mathrm{C}\right)$ & $-0,11$ & $-0,27$ & 0,09 & 0,16 & $-0,63$ & $0,81 * * *$ \\
\hline Longevidad foliar $(\mathrm{LF})^{+}$ & $-0,34$ & $-0,85 * * *$ & $-0,02$ & $-0,04$ & $-0,11$ & $-0,14$ \\
\hline Tiempo de inicio de crecimiento vegetativo (TICV) & $-0,10$ & $-0,25$ & $-0,40$ & $-0,71 * *$ & $-0,15$ & $-0,19$ \\
\hline Duración de crecimiento vegetativo (DCV) & $-0,10$ & $-0,25$ & $\mathbf{0 , 5 1}$ & $0,91 * * *$ & 0,04 & 0,05 \\
\hline Tasa de crecimiento relativo de la rama $(\mathrm{TCR})^{+}$ & 0,19 & 0,47 & $-0,48$ & $-0,85 * * *$ & 0,03 & $-0,03$ \\
\hline
\end{tabular}

$\left(^{+}\right)$Variable transformada a logaritmo natural. Las variables con mayor peso en cada componente principal se destacan con negrilla. Coeficientes de correlación de Pearson $(r)$ muestran la correlación de las variables originales con cada componente principal. Niveles de significancia para esas correlaciones: $P<0,05(*) ; P<0,01(* *) ; P<0,001(* * *)$. 

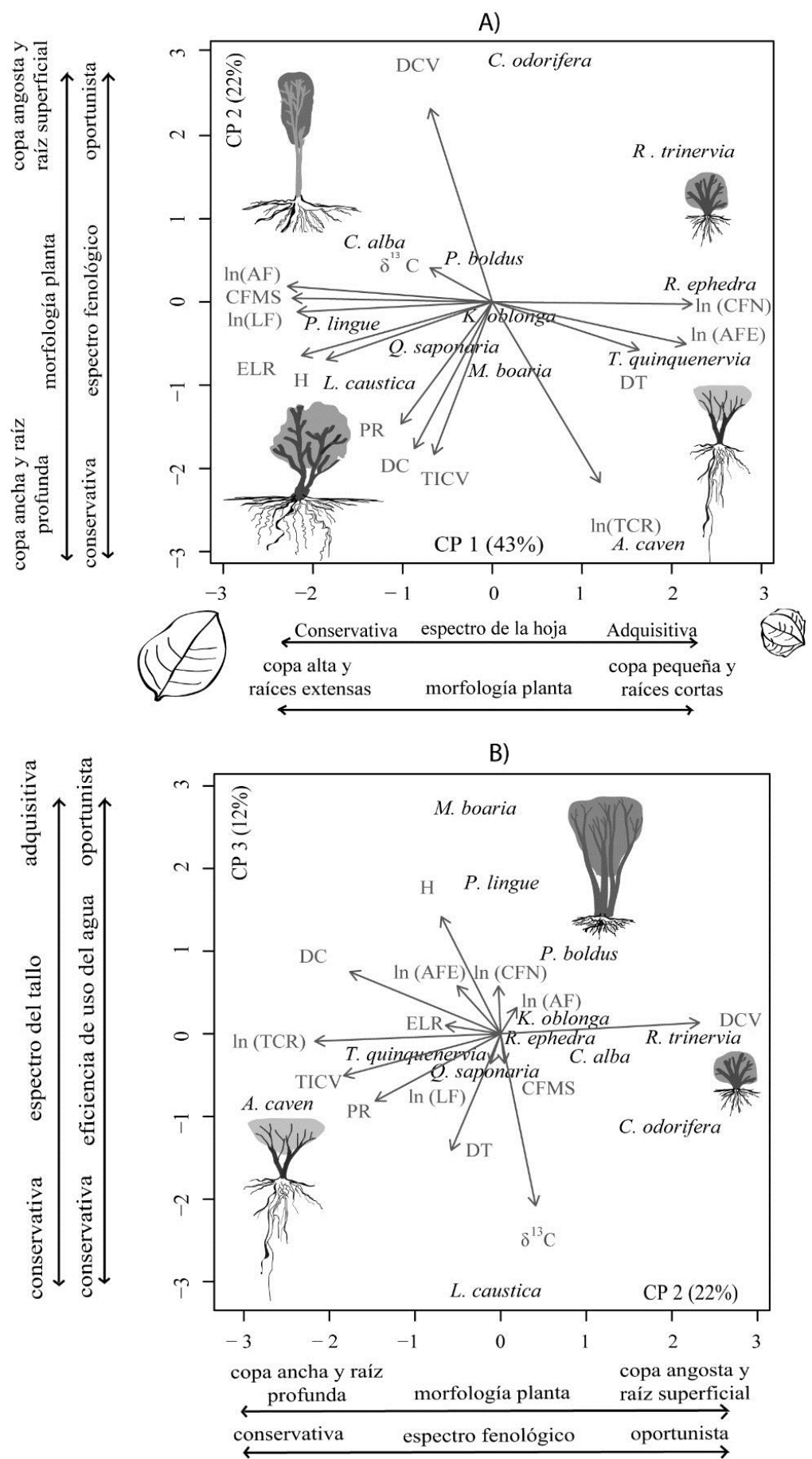

Figura 1. Análisis de componentes principales mostrando las múltiples dimensiones ecológicas de coordinación de 14 rasgos funcionales a través de 12 especies leñosas dominantes del matorral de Chile. A) El eje 1 muestra el espectro de la economía de la hoja acoplado a la morfología de la planta (altura de planta y extensión lateral de raíces); el eje 2 muestra el espectro de la fenología acoplado a la morfología de la planta (diámetro de copa y profundidad de raíces. B) El eje 3 muestra el espectro de la economía del tallo y de la eficiencia de uso del agua.

Principal component analysis showing the different dimensions of functional trait coordination across 12 dominant woody species of the Chilean matorral. A) The axis 1 shows the leaf economic spectrum coupled with the plant size and root lateral extension; the axis 2 shows the phenology spectrum associated with rooting depth and crown width. B) The axis 3 exhibits, the wood economic spectrum and water use efficiency. 
ción de crecimiento, mayor tasa de crecimiento, raíces profundas y diámetros de copa anchas (cuadro 4, figura 1A).

Finalmente, el componente principal tres explicó el $12 \%$ de la variabilidad y mostró las diferencias de las especies respecto a su tolerancia a la sequía. Por un lado, separó a las especies más resistentes a la sequía que combinaron mayor densidad del tallo (relacionada con una menor capacidad de transporte del agua) y mayor composición isotópica de ${ }^{13} \mathrm{C}$ de la hoja (relacionada positivamente con mayor eficiencia intrínseca del uso de agua). Por otro lado, separó a las especies oportunistas para el uso del agua que combinaron atributos opuestos (cuadro 4, figura 1B).

El primer componente principal separó significativamente a las especies esclerófilas y malacófilas $(F=28,3$; $P<0,001$ ) (figura $2 \mathrm{~A}$ ). En contraste, el segundo y tercer componente principal no separaron significativamente a las esclerófilas y malacófilas $(P=0,60$ y $P=0,49$, respectivamente), sugiriendo que en esas últimas dimensiones funcionales no hubo segregación de especies entre esos grupos funcionales (figura $2 \mathrm{~B} \mathrm{y} \mathrm{C}$ ).

\section{DISCUSIÓN}

Las especies leñosas esclerófilas y malacófilas del matorral mediterráneo de Chile no difieren totalmente en sus estrategias de uso de los recursos a través de una única dimensión ecológica que las contrasta como conservativas o adquisitivas. Las especies esclerófilas y malacófilas coordinan sus estrategias de uso de los recursos a través de múltiples dimensiones ecológicas, en las cuales las especies pueden combinar o no valores de rasgos conservativos y adquisitivos.

Las especies esclerófilas y malacófilas solamente presentaron diferencias significativas en algunos rasgos morfológicos de la planta (altura máxima de la planta, extensión lateral de raíces) y de hoja (área foliar específica, contenido de materia seca de la hoja, contenido de nitrógeno entre otros), pero no difirieron en la profundidad

de raíces, densidad de tallo, composición isotópica de ${ }^{13} \mathrm{C}$ $\mathrm{y}$ atributos fenológicos como sugirieron previos estudios en el matorral (Mooney y Dunn 1970, Montenegro et al. 1979, Canadell y Zedler 1994). Los valores de rasgos funcionales de uso de los recursos de las especies coordinaron través de tres dimensiones ecológicas: a) el espectro de la economía de la hoja acoplado con el espectro de la morfología de la planta (altura máxima de la planta y extensión lateral de raíces), b) el espectro fenológico (inicio de crecimiento, duración de crecimiento y tasa de crecimiento) acoplado con la profundidad de raíces y el diámetro de copa, y c) el espectro de la eficiencia de uso del agua a nivel de la hoja acoplado con el espectro de la economía de la madera, representado por la densidad de la madera. La variación de los rasgos funcionales a través de múltiples dimensiones coincide con estudios que observaron una débil coordinación entre rasgos funcionales relacionados con el uso de los recursos a través de especies leñosas en bosques tropicales húmedos (Baraloto et al. 2010, Fortunel et al. 2012), bosques tropicales semi-áridos (Silva et al. 2018), chaparrales de clima tipo mediterráneo de California (Ackerly 2004) y desiertos (Delpiano et al. 2020). Así, las estrategias de uso de los recursos en el matorral de Chile no coordinarían a través de una única dimensión ecológica como fue sugerido por estudios a escala local en bosques Mediterráneos de España (Riva et al. 2016) y estudios realizados a escala regional y global a través de especies de plantas con diferentes formas de vida (Westoby et al. 2002, Díaz et al. 2004).

La primera dimensión ecológica sugiere la existencia de un espectro de economía de la hoja que refleja los tra$d e$-offs de las especies para adquirir o conservar el carbono. Las especies esclerófilas a nivel de hoja combinaron valores de rasgos mayoritariamente conservativos, tales como mayor contenido foliar de materia seca, menor área foliar específica, menor contenido de nitrógeno y mayor longevidad, mientras que las especies malacófilas combi-

B)

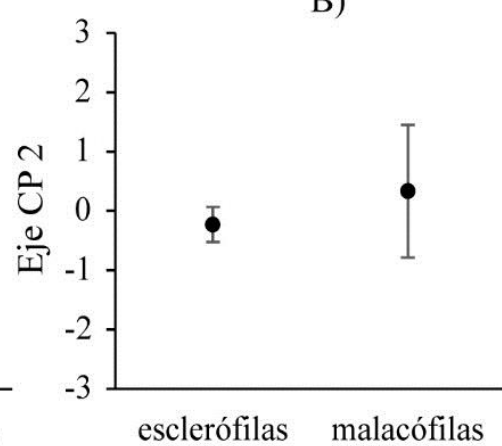

esclerofilas malacófilas
A)

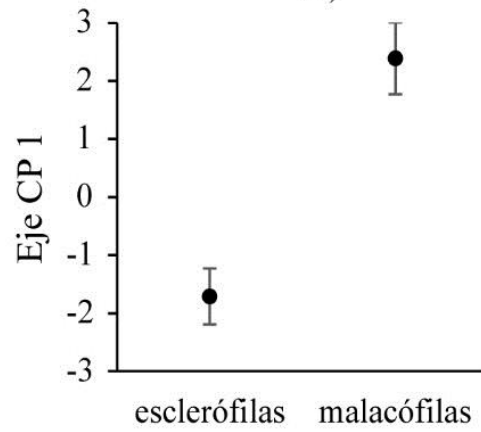

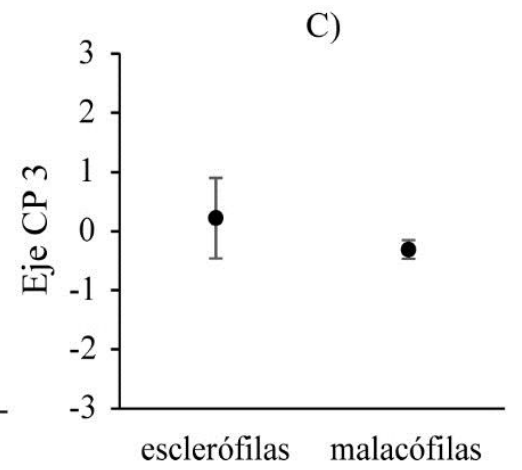

Figura 2. Análisis de varianza de una vía para evaluar la segregación de rasgos funcionales de especies esclerófilas y malacófilas a través de los tres ejes del ACP.

Analysis of variance (one way) evaluating segregation between sclerophyllous and malacophyllous species across three axis of the principal component analysis (CP1, CP2 and CP3). 
naron valores de rasgos foliares opuestos que sugieren una estrategia adquisitiva. Este patrón de coordinación a nivel de hoja coincide con previos estudios realizados entre especies de bosques mediterráneos a escala local (Mooney y Dunn 1970, Ackerly 2004, Prieto et al. 2018) y entre especies de diferentes formas de vida a escala global (Wright et al. 2004, Reich 2014, Diaz et al. 2016).

Esta primera dimensión ecológica también fue conformada por un espectro de la morfología de la planta donde coordinaron la altura máxima de la planta y a la extensión lateral de raíces. Este patrón de coordinación refleja los trade-offs que tienen las especies para capturar los recursos de luz y agua del suelo. Las especies esclerófilas combinaron mayor altura de máxima de planta y mayor extensión lateral de raíces, lo que exhibe su mayor habilidad competitiva para capturar los recursos del suelo y la luz (Grime 2001, Pérez-Harguindeguy et al. 2013); mientras que las especies malacófilas combinaron valores de rasgos opuestos, lo que refleja su menor habilidad competitiva para capturar recursos y su mayor tolerancia a la sombra (Zhao y Yan 2016). La coordinación del espectro de la economía de la hoja y algunos rasgos morfológicos de la planta en una misma dimensión coincide con estudios realizados a escala local que sugieren una coordinación entre rasgos de hoja y copa entre especies leñosas de bosques mediterráneos (Ackerly 2004, de la Riva et al. 2016) y bosques subtropicales (Zhao et al. 2016). Sin embargo, este patrón de coordinación no coincide con algunos estudios realizados a escala local y regional que muestran que el espectro de economía de la hoja es independiente de la morfología de la planta, del tallo y raíz (Díaz et al. 2004, 2016, Baraloto et al. 2010, Fortunel et al. 2012).

La primera dimensión muestra que las especies no combinaron valores de rasgos funcionales de una única estrategia de uso de los recursos como sugiere el espectro de la economía de la planta (Reich 2014). Las esclerófilas combinaron algunos valores de rasgos conservativos para usar el carbono (por ejemplo, mayor contenido de materia seca de la hoja, menor área foliar específica) y valores de rasgos adquisitivos (por ejemplo, mayor área foliar, altura de planta, extensión lateral de raíces y menor densidad de madera), mientras que las especies malacófilas combinaron rasgos con valores opuestos. Este patrón de coordinación contrasta con aquellos encontrados en ambientes mediterráneos de la Cuenca del Mediterráneo de España, donde los valores de rasgos funcionales de especies esclerófilas y malacófilas convergen coordinadamente en una única estrategia conservativa o adquisitiva, respectivamente. Por ejemplo, en esos ecosistemas las especies esclerófilas comparadas con las malacófilas exhiben una estrategia conservativa con una menor altura de planta, menor área foliar específica, mayor eficiencia de uso del agua, mayor densidad de tallo y menor longitud específica de la raíz, mientras que las malacófilas deciduas combinan valores de rasgos opuestos (Pérez-Ramos et al. 2012, de la Riva et al. 2016, Prieto et al. 2018, Querejeta et al. 2018).
En los matorrales de Chile, este patrón de coordinación en la primera dimensión ecológica podría sugerir que las especies esclerófilas tienen una lenta adquisición de carbono, pero una mayor habilidad competitiva para interceptar luz y para capturar agua superficial y nutrientes del suelo, mientras que las especies malacófilas tienen una rápida asimilación de carbono, pero mayores limitaciones para competir por la luz, crecer y capturar los recursos superficiales del suelo (Mooney y Dunn 1970). Posiblemente, esas características permitan a las especies esclerófilas y malacófilas usar los recursos a través de ambientes con diferente disponibilidad de luz y/o nutrientes (Domínguez et al. 2012). Por un lado, las especies esclerófilas del matorral podrían estar adaptadas para crecer en ambientes con menor radiación solar y mayor disponibilidad de agua, como en laderas de menor exposición a la radiación solar y/o limitados por nutrientes (Mooney y Dunn 1970), mientras las malacófilas podrían dominar condiciones ambientales opuestas (Armesto y Martinez 1978).

La variación y coordinación de los rasgos de profundidad de raíces, diámetro de copa y la fenología formaron una segunda dimensión ecológica (independiente de la primera) que reflejaría los trade-offs de las especies para adquirir los recursos a través de diferentes temporadas del año y diferentes profundidades del suelo debido a la alta irregularidad espacial (a través de horizontes en el suelo) y temporal en la disponibilidad de agua y nutrientes en ecosistemas mediterráneos (Mooney y Dunn 1970). Por un lado, las especies que combinaron valores de rasgos conservativos, tales como raíces profundas, inicio de crecimiento tardío, menor duración de crecimiento, mayor tasa de crecimiento y mayor diámetro de copa podrían crecer en condiciones climáticas óptimas con mayor disponibilidad de recursos y durante periodos breves (Wolkovich y Cleland 2014). Por ejemplo, las especies con un inicio tardío de crecimiento podrían mantenerse en reposo durante la temporada de lluvia y evitar las bajas temperaturas del invierno austral (Montenegro et al. 1979). Además, las especies con raíces profundas podrían esperar a que el agua de las precipitaciones infiltre y recargue el suelo a mayores profundidades para usarlas sin tener incertidumbre sobre su disponibilidad (Canadell y Zedler 1994). En adición, las especies con una copa de mayor diámetro desplegarían hojas menos sombreadas interceptando mayor radiación y alcanzando mayores tasas de fotosíntesis (Poorter et al. 2012), la cual combinada con una mayor tasa de crecimiento permitiría a las especies crecer aceleradamente para fijar los productos de la fotosíntesis y evitar exponerse a las altas temperaturas y la alta radiación durante verano (Mooney y Dunn 1970). Por otro lado, las especies que combinaron valores de rasgos funcionales adquisitivos, tales como raíces superficiales, un inicio de crecimiento temprano, mayor duración de crecimiento, menor tasa de crecimiento y menor diámetro de copa podrían usar los recursos bajo condiciones ambientales temporalmente marginales y por largos perio- 
dos (Chuine 2010). Por ejemplo, las especies de raíces superficiales y de inicio temprano de crecimiento tendrían una rápida adquisición del agua y nutrientes durante los primeros eventos de precipitación, incluso bajo riesgo de heladas (Canadell y Zedler 1994, Moreno-Gutiérrez et al. 2012). Además, una mayor duración de crecimiento y una lenta tasa de crecimiento permitiría a las especies capturar mayor cantidad de recursos y completar la fijación de los productos de la fotosíntesis durante invierno y primavera (Castro-Díez et al. 2003).

Una tercera dimensión ecológica formada por la variación y coordinación de la composición isotópica de ${ }^{13} \mathrm{C}$ de la hoja y la densidad de la madera sugiere que las especies independientemente de las características morfológicas de la hoja para adquirir carbono, tamaño de planta, profundidad de raíz y fenología pueden desarrollar diferentes estrategias para usar el agua. Este desacople de la composición isotópica foliar de ${ }^{13} \mathrm{C}$ respecto al espectro de la economía de la hoja y otras dimensiones funcionales fue también encontrado entre especies del chaparral de California (Ackerly 2004) y en bosques mediterráneos de España (Méndez-Alonzo et al. 2019). Posiblemente, este patrón de coordinación refleja los "trade-offs" que tienen las especies del matorral para usar el agua a través de diferentes condiciones de disponibilidad de agua en los horizontes superficiales del suelo debido a la alta heterogeneidad estructural y topográfica los suelos en el matorral (Rundel et al. 2016). Por ejemplo, las especies que combinan una mayor densidad de madera y mayor eficiencia de uso del agua (valores menos negativos de composición isotópica ${ }^{13} \mathrm{C}$ ) podrían reflejar una estrategia conservativa de uso del agua en suelos con alto déficit hídrico, la cual permitiría incrementar la resistencia del tallo para el transporte de agua, lo que consecuentemente podría reducir el potencial hídrico en la hoja, la conductancia estomática y las tasas de transpiración. Al contrario, las especies con una combinación de valores opuestos de esos rasgos podrían reflejar una estrategia oportunista de uso del agua adaptada a suelos con mayor disponibilidad hídrica, la cual permitiría aumentar la conductividad hidráulica del tallo para transportar el agua, la apertura estomática para el intercambio gaseoso en la hoja, la transpiración y en consecuencia reducir la eficiencia de uso del agua en la hoja, pero al costo de aumentar su riesgo al embolismo (Moreno-Gutiérrez et al. 2012, Apgaua et al. 2015).

En el presente estudio los valores de rasgos funcionales a través de toda la planta no convergieron hacia una única estrategia ecológica de uso de recursos como una respuesta a las restricciones ambientales que deberían afectar similarmente a toda la planta (Reich 2014). En este estudio, las especies del matorral combinaron valores de rasgos adquisitivos y valores de rasgos conservativos; y además formaron diferentes dimensiones de coordinación de rasgos funcionales. Posiblemente, los factores abióticos y bióticos variando en el espacio (arriba o abajo del suelo) y en el tiempo operan con diferente intensidad y dirección filtrando rasgos funcionalmente contrastantes (Weemstra et al. 2016). Este estudio es uno de los primeros evaluando la variación y coordinación rasgos funcionales de diferentes órganos de las plantas leñosas en bosques mediterráneos, incluyendo la coordinación del espectro de la economía de la hoja, con rasgos morfológicos de la planta, tallo, raíz y fenología. Sin embargo, sugerimos considerar que futuros estudios incluyan mayor número de rasgos funcionales de raíz (por ejemplo, longitud específica de la raíz, densidad de la raíz, contenido de nitrógeno) y rasgos del tallo (diámetro de vasos, densidad de vasos, contenido de materia seca del tallo), los cuales podrían permitir una evaluación más completa de las estrategias de uso de los recursos de las especies del matorral de Chile.

\section{CONCLUSIONES}

Las especies esclerófilas y malacófilas no difieren completamente a través de diferentes órganos de la planta y la fenología. Además, los patrones de variación y coordinación de rasgos funcionales a través de las especies sugieren que existe un desacople de estrategias de uso de los recursos en múltiples dimensiones. Las especies esclerófilas y malacófilas tienen diferencias solo en una dimensión de las tres observadas, específicamente en la primera dimensión que está relacionada al espectro de la economía de la hoja y los rasgos morfológicos de altura máxima de planta y la extensión lateral de raíz. Las especies esclerófilas y malacófilas no reflejan diferentes estrategias a través de otras dimensiones ecológicas relacionadas con su fenología y tolerancia a la sequía. Por tanto, existen múltiples dimensiones de coordinación de rasgos funcionales que reflejan las estrategias contrastantes de las especies del matorral para usar los recursos. Este estudio muestra la necesidad de usar múltiples rasgos funcionales para explicar las estrategias de las especies para usar los recursos en ecosistemas mediterráneos y predecir procesos ecológicos en el matorral de Chile.

\section{AGRADECIMIENTOS}

Los autores agradecen al CONICYT por beneficiarnos con la beca de doctorado de Ariel Isaías Ayma Romay y el financiamiento FONDECYT N ${ }^{\circ} 1150877$. Agradecemos infinitamente las evaluaciones, comentarios y sugerencias de los profesores Javier Simonetti, Aurora Gaxiola, Edmundo Acevedo y Lohengrin Cavieres durante el desarrollo del proyecto de doctorado. También agradecemos a la Dra. Aurora Gaxiola por proporcionarnos su base de datos no publicada de contenido de nitrógeno de la hoja de especies leñosas del bosque esclerófilo, la cual fue complementada con datos de literatura para propósitos de nuestro estudio. También agradecemos a María Andrea Díaz y Francisca Herrera quienes colaboraron en el trabajo de campo y laboratorio para la medición de rasgos funcionales de las plantas. 


\section{REFERENCIAS}

Ackerly D. 2004. Functional strategies of chaparral shrubs in relation to seasonal water deficit and disturbance. Ecological Monographs 74(1):25-44. DOI: https://doi.org/10.1890/03-4022

Apgaua DMG, FY Ishida, DYP Tng, MJ Laidlaw, R Santos, R Rumman, D Eaumus, J Hotum, SGW Laurance. 2015. Functional traits and water transport strategies in lowland tropical rainforest trees. PLOS ONE 10(6):1-19. DOI: https://doi.org/10.1371/journal.pone.0130799

Armesto J, JA Martinez, 1978. Relations between vegetation structure and slope aspect in the mediterranean region of Chile. Journal of Ecology 66: 881-889.

Baraloto C, CE Paine, L Poorter, J Beauchene, D Bonal, AM Domenach, B Hérault, S Patiño, JC Roggy, J Chave, 2010. Decoupled leaf and stem economics in rain forest trees. Ecology Letters 13, 1338-1347. DOI: https://doi.org/10.1111/ j.1461-0248.2010.01517.x

Bown HE, JP Fuentes, JF Perez-Quezada, N Franck. 2014. Soil respiration across a disturbance gradient in sclerophyllous ecosystems in Central Chile. Ciencia e Investigación Agraria 41(1):89-106. DOI: https://doi.org/10.4067/S071816202014000100009

Canadell J, P Zedler. 1994. Underground structures of woody plants in Mediterranean ecosystems of Australia, California and Chile. In Arroyo MK, P Zedler, M Fox eds. Ecology and biogeography of mediterranean ecosystems in Chile, California and Australia. New York, USA. Springer - Verlag. p. 177-210.

Carvajal DE, AP Loayza, RS Rios, CA Delpiano, FA Squeo. 2019. A hyper-arid environment shapes an inverse pattern of the fast-slow plant economics spectrum for above-, but not below-ground resource acquisition strategies. Journal of Ecology 107: 1079-1092. DOI: https://doi. org/10.1111/1365-2745.13092

Castro-Díez P, G Montserrat-Martí, JHC Cornelissen. 2003. Trade-Offs between phenology, relative growth rate, life form and seed mass among 22 Mediterranean woody species. Plant Ecology 166:117-129. DOI: https://doi. org/10.1023/A:1023209230303

Chave J, D Coomes, S Jansen, SL Lewis, NG Swenson, AE Zanne. 2009. Towards a worldwide wood economics spectrum. Ecology Letters 12(4):351-366. DOI: https://doi. org/10.1111/j.1461-0248.2009.01285.x

Chuine I. 2010. Why does phenology drive species distribution? Philosophical Transactions of the Royal Society B: Biological Sciences 365(1555):3149-3160. DOI: https://doi. org/10.1098/rstb.2010.0142

de la Riva EG, A Tosto, IM Pérez-Ramos, CM Navarro-Fernández, M Olmo, NPR Anten, T Marañon, Villar, R. 2016. A plant economics spectrum in Mediterranean forests along environmental gradients: is there coordination among leaf, stem and root traits? Journal Vegetation Science 27: 187199. DOI: https://doi.org/10.1111/jvs.12341

Delpiano CA, I Prieto, AP Loayza, DE Carvajal, FA Squeo. 2020. Different responses of leaf and root traits to changes in soil nutrient availability do not converge into a communitylevel plant economics spectrum. Plant Soil 450: 463-478. DOI: https://doi.org/10.1007/s11104-020-04515-2

Díaz S, J Hodgson, K Thompson, M Cabido, J Cornelissen, A Jalili, G Montserrat-Martí, JP Grime, F Zarrinkamar, Y
Asri, SR Band, S Basconcelo, P Castro-Díez, G Funes, B Hamzehee, M Khoshnevi, N Pérez-Harguindeguy, MC Pérez-Rontomé, A Shirvany, F Vendramini, S Yazdani, R Abbas-Azimi, A Bogaard, S Boustani, M Charles, M Dehghan, L de Torres-Espuny, V Falczuk, J GuerreroCampo, A Hynd, G Jones, E Kowsary, F Kazemi-Saeed, M Maestro-Martínez, A Romo-Díez, S Shaw, B Siavash, P Villar-Salvador, MR Zak. 2004. The plant traits that drive ecosystems: Evidence from three continents. Journal of Vegetation Science 15(3):295-304. DOI: https://doi. org/10.1111/j.1654-1103.2004.tb02266.x

Díaz S, J Kattge, JHC Cornelissen, I Wright, S Lavorel, S Dray, B Reu, M Kleyer, C Wirth, IC Prentice, E Garnier, G Bönisch, M Westoby, H Poorter, P Reich, AT Moles, J Dickie, AN Gillison, AE Zanne, S Pierce, B Shipley, D Kirkup, F Casanoves, JS Joswig, A Günther, V Falczuk. 2016. The global spectrum of plant form and function. Nature 529, 167-171. DOI: https://doi.org/10.1038/nature16489

Domínguez, MT, C Aponte, IM Pérez-Ramos, LV García, R Villar, T Marañón. 2012. Relationships between leaf morphological traits, nutrient concentrations and isotopic signatures for Mediterranean woody plant species and communities. Plant Soil 357: 407-424. DOI: https://doi.org/10.1007/ s11104-012-1214-7

Fortunel C, PVA Fine, C Baraloto. 2012. Leaf, stem and root tissue strategies across 758 Neotropical tree species. Functional Ecology 26 (5):1153-1161. DOI: https://doi. org/10.1111/j.1365-2435.2012.02020.x

Grime JP. 2001. Plant strategies, vegetation processes, and ecosystem properties. New York, USA, Wiley \& Sons. 410 p.

Lima, ALA, MJN Rodal. 2010. Phenology and wood density of plants growing in the semi-arid region of northeastern Brazil. Journal of Arid Environments 74: 1363-1373. DOI: https://doi.org/10.1016/j.jaridenv.2010.05.009

Méndez-Alonzo, R, FW Ewers, AL Jacobsen, RB Pratt, C Scoffoni, MK Bartlett, L Sack. 2019. Covariation between leaf hydraulics and biomechanics is driven by leaf density in Mediterranean shrubs. Trees 33: 507-519. DOI: https:// doi.org/10.1007/s00468-018-1796-7

Montenegro G, ME Aljaro, J Kummerow. 1979. Growth dynamics of Chilean matorral shrubs. Botanical Gazette 140(1):114-119.

Montenegro G, G Avila, ME Aljaro, R Osorio, M Gomez. 1989. Chile. In Orshan G ed. Plant pheno-morphological studies in mediterranean type ecosystems. Dordrencht, The Netherlands, Kluwer Academic Publishers. p. 347-387.

Mooney H, EL Dunn. 1970. Convergent evolution of mediterraneanclimate evergreen sclerophyll shrubs. Evolution 24(2):292303. DOI: https://doi.org/10.1111/j.1558-5646.1970.tb01762.x

Moreno-Gutiérrez C, T Dawson, E Nicolás, J Querejeta. 2012. Isotopes reveal contrasting water use strategies among coexisting plant species in a mediterranean ecosystem. New Phytologist 196(2):489-496. DOI: https://doi.org/10.1111/ j.1469-8137.2012.04276.x

Pérez-Harguindeguy N, S Díaz, E Garnier, S Lavorel, L Poorter, P Jaureguiberry, MS Bret-Harte, WK Cornwell, JM Craine, DE Gurvich, C Urcelay, EJ Veneklaas, P Reich, L Poorter, IJ Wright, P Ray, L Enrico, JG Pausas, AC de Vos, N Buchmann, G Funes, F Quétier, JG Hodgson, K Thompson, HD Morgan, H ter Steege, L Sack, B Blonder, P Poschlod, MV Vaieretti, G Conti, AC Staver, S Aquino, JHC Cornelissen. 
2013. New handbook for standardised measurement of plant functional traits worldwide. Australian Journal of Botany 61(3):167-234. DOI: https://doi.org/10.1071/BT12225_CO

Pérez-Ramos, IM., C Roumet, P Cruz, A Blanchard, P Autran, E Garnier. 2012. Evidence for a "plant community economics spectrum" driven by nutrient and water limitations in a Mediterranean rangeland of southern France. Journal of Ecology 100: 1315-1327. DOI: https://doi.org/10.1111/1365-2745.12000

Poorter, L, Lianes, E, Moreno-de las Heras, M, Zabala, M. 2012. Architecture of Iberian canopy tree species in relation to wood density, shade tolerance and climate. Plant Ecology 213:707722. DOI: https://doi.org/10.1007/s11258-012-0032-6

Prieto, I, JI Querejeta, J Segrestin, F Volaire, C Roumet. 2018. Leaf carbon and oxygen isotopes are coordinated with the leaf economics spectrum in Mediterranean rangeland species. Functional Ecology 32: 612-625. DOI: https://doi. org/10.1111/1365-2435.13025

Querejeta, JI, I Prieto, P Torres, M Campoy, MM Alguacil, A Roldán. 2018. Water-spender strategy is linked to higher leaf nutrient concentrations across plant species colonizing a dry and nutrient-poor epiphytic habitat. Environmental and Experimental Botany 153: 302-310. DOI: https://doi. org/10.1016/j.envexpbot.2018.06.007

R Core Team, 2020. R: A language and environment for statistical computing.

Reich PB. 2014. The world-wide "fast-slow" plant economics spectrum: a traits manifesto. Journal of Ecology 102(2):275301. DOI: https://doi.org/10.1111/1365-2745.12211

Roumet C, M Birouste, C Picon-Cochard, M Ghestem, N Osman, S Vrignon-Brenas, K Cao, A Stokes. 2016. Root structure - function relationships in 74 species: evidence of a root economics spectrum related to carbon economy. New Forest 210: 815-826. DOI: https://doi.org/10.1111/nph.13828
Rundel, PW, MTK Arroyo, RM Cowling, JE Keeley, BB Lamont, P Vargas. 2016. Mediterranean biomes: Evolution of their vegetation, floras, and climate. Annual Review of Ecology, Evolution, and Systematics 47, 383-407. DOI: https://doi. org/10.1146/annurev-ecolsys-121415-032330

Silva, JLA, AF Souza, A Caliman, EL Voigt, JE Lichston. 2018. Weak whole-plant trait coordination in a seasonally dry South American stressful environment. Ecology and Evolution 8: 4-12. DOI: https://doi.org/10.1002/ece3.3547

van Buuren, S, K Groothuis-Oudshoorn. 2011. mice: Multivariate imputation by chained equations in R. Journal of Statistical Software 45, 1-67. DOI: https://doi.org/10.18637/jss. $\underline{\mathrm{v} 045.103}$

Weemstra M, L Mommer, EJ Visser, J Ruijven, T Kuyper, GMJ Mohren, F Sterck. 2016. Towards a multidimensional root trait framework: a tree root review. New Phytology 211: 1159-1169. DOI: https://doi.org/10.1111/nph.14003

Wolkovich EM, EE Cleland. 2014. Phenological niches and the future of invaded ecosystems with climate change. $A o B$ Plants 6: 1-16. DOI: https://doi.org/10.1093/aobpla/plu013

Wright IJ, PB Reich, M Westoby, D Ackerly, Z Baruch, F Bongers, J Cavender-Bares, T Chapin, JHC Cornelissen, M Diemer, J Flexas, E Garnier, PK Groom, J Gulias, K Hikosaka, B Lamont, T Lee, W Lee, C Lusk, J Midgley, M Navas, Ü Niinemets, J Oleksyn, N Osada, H Poorter, P Poot, L Prior, V Pyankov, C Roumet, SC Thomas, MG Tjoelker, EJ Veneklaas, R Villar. 2004. The worldwide leaf economics spectrum. Nature 428(6985): 821-827. DOI: https://doi. org/10.1038/nature02403

Zhao Y, A Ali, E Yan. 2016. The plant economics spectrum is structured by leaf habits and growth forms across subtropical species. Tree Physiology 37: 173-185. DOI: https://doi. org/10.1093/treephys/tpw098. 


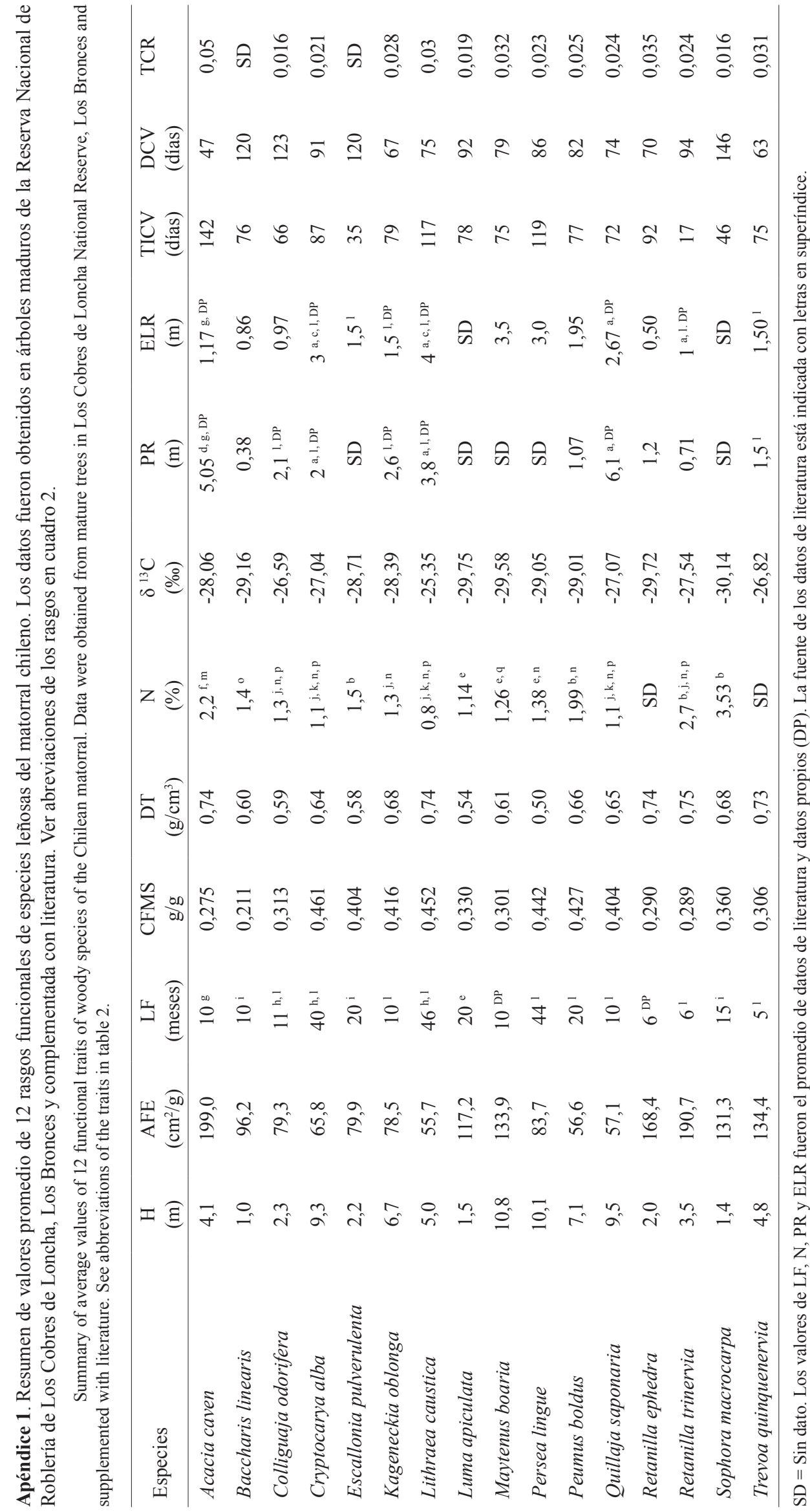


Apéndice 2. Resumen de rasgos funcionales obtenidos para el matorral de Chile.

Summary of functional traits obtained for the Chilean matorral.

\begin{tabular}{lccl}
\hline Rasgo & $\mathrm{N}^{\circ}$ plantas por especie & $\mathrm{N}^{\circ}$ de especies evaluadas & Fuente de datos \\
\hline Altura máxima de la copa $(\mathrm{m})$ & $6-56$ & 16 & propio \\
Diámetro de copa $(\mathrm{m})$ & $6-56$ & 16 & propio \\
Profundidad de raíz (m) & $2-17$ & 11 & propio y literatura \\
Extensión lateral de la raíz (m) & $2-17$ & 14 & propio y literatura \\
Área foliar $\left(\mathrm{cm}^{2}\right)$ & $6-20$ & 16 & propio \\
Área foliar específica $\left(\mathrm{cm}^{2} \mathrm{~g}^{-1}\right)$ & $6-20$ & 16 & propio \\
Contenido foliar de materia seca $\left(\mathrm{g} \mathrm{g}^{-1}\right)$ & $6-20$ & 16 & propio \\
Longevidad foliar (meses) & $3-5$ & 16 & literatura \\
Contenido foliar de nitrógeno (\%) & $3-5$ & 14 & literatura \\
Composición isotópica foliar ${ }^{13} \mathrm{C}$ & $3-30$ & 16 & propio \\
Densidad específica del tallo & $6-21$ & 16 & propio \\
Tiempo de inicio de crecimiento (días desde el 1/07/2017) & $3-10$ & 16 & propio \\
Duración del crecimiento vegetativo (días) & $3-10$ & 16 & propio \\
Tasa de crecimiento relativa (cm día $\left.{ }^{-1}\right)$ & $3-10$ & 14 & propio \\
\hline
\end{tabular}

Apéndice 3. Relación de la composición isotópica ${ }^{13} \mathrm{C}\left(\delta{ }^{13} \mathrm{C}\right)$, discriminación isotópica $(\Delta)$ y la eficiencia intrínseca de uso de agua (EUAi) para 16 especies del bosque esclerófilo de Chile.

Relationship of $13 \mathrm{C}$ isotopic composition $(\delta 13 \mathrm{C})$, isotopic discrimination $(\Delta)$ and intrinsic water use efficiency (EUAi) for 16 species of the sclerophyllous forest of Chile.

\begin{tabular}{|c|c|c|c|c|c|c|c|c|c|}
\hline \multirow[b]{2}{*}{ Especie } & \multicolumn{3}{|c|}{$\delta{ }^{13} \mathrm{C}$} & \multicolumn{3}{|c|}{$\Delta$} & \multicolumn{3}{|c|}{ EUAi } \\
\hline & Media & Mín. & Máx. & Media & Min & $\operatorname{Max}$ & Media & Min & Max \\
\hline A.caven & $-28,06$ & $-26,92$ & $-29,76$ & 20,23 & 19,07 & 21,94 & 71,20 & 83,31 & 53,22 \\
\hline B.linearis & $-29,16$ & $-28,39$ & $-31,58$ & 21,34 & 20,55 & 23,77 & 59,53 & 67,76 & 33,99 \\
\hline C.alba & $-27,04$ & $-24,60$ & $-30,51$ & 19,20 & 16,73 & 22,69 & 82,00 & 107,89 & 45,28 \\
\hline C.odorifera & $-26,59$ & $-24,46$ & $-28,74$ & 18,74 & 16,59 & 20,91 & 86,84 & 109,37 & 63,98 \\
\hline E.pulverulenta & $-28,71$ & $-26,98$ & $-30,23$ & 20,88 & 19,13 & 22,41 & 64,35 & 82,70 & 48,19 \\
\hline K.oblonga & $-28,39$ & $-27,20$ & $-29,58$ & 20,55 & 19,36 & 21,76 & 67,73 & 80,31 & 55,10 \\
\hline L.apiculata & $-29,75$ & $-27,33$ & $-30,81$ & 21,92 & 19,49 & 23,00 & 53,37 & 78,94 & 42,05 \\
\hline L.caustica & $-25,35$ & $-22,43$ & $-29,73$ & 17,49 & 14,55 & 21,91 & 99,91 & 130,87 & 53,51 \\
\hline M.boaria & $-29,58$ & $-28,18$ & $-31,79$ & 21,75 & 20,34 & 23,98 & 55,13 & 69,94 & 31,73 \\
\hline P.boldus & $-29,01$ & $-26,62$ & $-31,47$ & 21,18 & 18,77 & 23,66 & 61,20 & 86,49 & 35,11 \\
\hline P.lingue & $-29,05$ & $-26,52$ & $-30,93$ & 21,22 & 18,67 & 23,11 & 60,72 & 87,55 & 40,86 \\
\hline Q.saponaria & $-27,07$ & $-24,62$ & $-30,07$ & 19,23 & 16,75 & 22,25 & 81,67 & 107,67 & 49,95 \\
\hline R.ephedra & $-29,72$ & $-29,53$ & $-29,91$ & 21,90 & 21,70 & 22,09 & 53,63 & 55,66 & 51,61 \\
\hline R.trinervia & $-26,09$ & $-24,93$ & $-27,93$ & 18,23 & 17,07 & 20,10 & 92,13 & 104,39 & 72,56 \\
\hline S.macrocarpa & $-30,14$ & $-28,44$ & $-32,25$ & 22,31 & 20,60 & 24,45 & 49,24 & 67,25 & 26,82 \\
\hline T.quinqunervia & $-26,82$ & $-25,86$ & $-27,56$ & 18,97 & 18,01 & 19,72 & 84,42 & 94,52 & 76,51 \\
\hline
\end{tabular}


La discriminación isotópica $(\Delta)$ corresponde (Ferrio et al. 2005):

$$
\Delta=\frac{\delta_{\text {atmósfera }}-\delta_{\text {planta }}}{1+(\delta|| \text { planta } / 1000)}
$$

Donde, $\delta_{\text {atmósfera }}$ corresponde a $-8 \%$ y $\delta_{\text {planta }}$ corresponde al valor de la muestra (en este caso el valor promedio de $\delta_{\text {planta }}$ para cada especie). Acorde a este modelo la relación entre la $\delta{ }^{13} \mathrm{C}$ y la $\Delta$ es linealmente inversa. La $\Delta$ representa el fraccionamiento de la $\delta{ }^{13} \mathrm{C}$ de la atmósfera a través de los procesos físicos y bioquímicos del proceso de fotosíntesis. De esta manera una mayor discriminación de moléculas de ${ }^{13} \mathrm{CO}_{2}$ durante la fotosíntesis refleja una mayor síntesis de moléculas de ${ }^{12} \mathrm{CO}_{2}$.

$\mathrm{La} \Delta$ tiene una relación lineal positiva con las proporciones de carbono intercelular en la hoja y el carbono de la

\section{REFERENCIAS}

Farquhar, GD, JR Ehleringer, KT Hubick. 1989. Carbon isotope discrimination and photosynthesis. Annu. Rev. Plant Physiol. Plant Mol. Biol. 40: 503-537. DOI: https://doi. org/10.1146/annurev.pp.40.060189.002443

Ferrio JP, V Resco, DG Williams, L Serrano, J Voltas, 2005. Stable isotopes in arid and semi-arid forest systems. Investigación Agraria. Sistemas y Recursos Forestales. 14: 371. DOI: https://doi.org/10.5424/srf/2005143-00929

Gaxiola, A (no publicado). Datos de contenido de nitrógeno foliar de especies leñosas del matorral de Chile.

Giliberto J, H Estay. 1978. Water stress in some chilean shrubs. Botanical Gazette 139:236-240.

Hoffmann A, J Kummerow. 1978. Root studies in the Chilean matorral. Oecologia 69:57-69. DOI: https://doi.org/10.1007/ $\underline{\mathrm{BF} 00344689}$

Kraus T, C Bianco, F Weberling. 2003. Root system morphology of Fabaceae species from central Argentina. Wulfenia 10: 61-72

Lusk CH, I Wright, P Reich. 2003. Photosynthetic differences contribute to competitive advantage of evergreen angiosperm trees over evergreen conifers in productive habitats. New Phytologist 160(2):329-336. DOI: https://doi. org/10.1046/j.1469- 8137.2003.00879.x.

Martinez I, E Zagal, C Ovalle, M Couteaux, NB Stolpe, N Valderrama. 2010. Litter decomposition of Acacia caven (Molina) and Lolium multiflorum Lam. in mediterrranean climate ecosystems. Chilean Journal Agricultural Research 70(3):454-464. DOI: https://doi.org/10.4067/S0718-58392010000300013

Montenegro G, R Gatti, F Riveros. 1976. Modificaciones foliares en Acacia caven (Mol.) Hook. et Am. por efecto de la contaminación ambiental. Medio Ambiente 2: 29-34

Montenegro G, ME Aljaro, A Walkowiak, R Saenger. 1982. Seasonality, growth and net productivity of herbs and shrubs of the Chilean matorral. In E Conrad, W Oechel eds. Symposium on dynamics and management of mediterranean-type ecosystems. San Diego, USA. Forest Service. p. 135-141.

Montenegro G, G Avila, ME Aljaro, R Osorio, M Gomez. 1989. Chile. In Orshan G ed. Plant pheno-morphological studies in mediterranean type ecosystems Dordrencht, The Netherlands, Kluwer Academic Publishers. p. 347-387. atmósfera $\left(\mathrm{c}_{\mathrm{i}} / \mathrm{c}_{\mathrm{a}}\right)$ (Farquhar et al. 1989). Asumiendo esta relación, la $\Delta$ se utiliza para calcular la eficiencia de uso del agua intrínseca $(\mathrm{EUAi}=$ Fotosíntesis/conductancia estomática) de la siguiente manera (Seibt et al. 2008):

$$
\mathrm{EUAi}=\frac{c_{a}}{1.6} \times \frac{b-\Delta}{b-a}
$$

Donde, $\mathrm{b}=27 \%$ es la discriminación de moléculas de ${ }^{13} \mathrm{CO}_{2}$ por parte de la Ribulosa bifosfato, y a $=4.4 \%$, es la discriminación estomática de moléculas de ${ }^{13} \mathrm{CO}_{2}$ en el proceso de intercambio gaseoso (O'Leary 1988).

Debido a que la $\Delta$ y la EUAi tienen una relación lineal y su estimación tiene de base a la $\delta{ }^{13} \mathrm{C}$, resulta que este último tiene una relación lineal positiva y puede usarse como un indicador de EUAi (Pérez-Harguindeguy et al. 2013).

Mooney H. 1977. Convergent evolution in Chile and California. Pensylvania, Dowden Hutchinson \& Ross. 219 p.

Morini C. 2013. Estimación de la fotosíntesis, conductancia estomática y transpiración en Quillaja saponaria, Cryptocarya alba y Lithraea caustica en la Reserva Nacional Roblería de Los Cobres de Loncha, Región Metropolitana. Tesis Ingeniero Forestal. Santiago, Chile. Facultad de Ciencias Forestales y Conservación de la Naturaleza, Universidad de Chile. 65 p.

O'Leary M. 1988. Carbon isotopes in photosynthesis. BioScience 38(5):328-336

Orshan G, G Montenegro, G Avila, ME Aljaro, A Walckowiak, AM Mujica. 1984. Plant growth forms of Chilean matorral a monocharacter growth form analysis along an altitudinal transect from sea level to $2000 \mathrm{~m}$ a. s. 1. Bulletin de la Société Botanique de France 131:411-425. DOI: https://doi. org/10.1080/01811789.1984.10826681

Pérez-Harguindeguy N, CM Blundo, DE Gurvich, S Díaz, E Cuevas. 2008. More than the sum of its parts? Assessing litter heterogeneity effects on the decomposition of litter mixtures through leaf chemistry. Plant and Soil 303(1-2):151159. DOI: https://doi.org/10.1007/s11104-007-9495-y

Rundel P. 1981. The matorral zone of Central Chile. In di Castri F, FD Goodall eds. Mediterranean-type shrublands. New York, USA. Elsevier. p. 175-201.

Saiz, F, C Ñunez. 2000. Aspectos ecológicos de las cecidas del Norte Árido de Chile (Segunda Región). Revista Chilena de Historia Natural 26:41-51

Satti P, M Mazzariano, M Gobbi, F Funes, L Roselli, H Fernández. 2003. Soil $\mathrm{N}$ dynamics in relation to leaf litter quality and soil fertility in north-western Patagonian forests. Journal of Ecology 91: 173-181.

Seibt U, A Rajabi, H Griffiths, JA Berry. 2008. Carbon isotopes and water use efficiency: sense and sensitivity. Oecologia 155: 441-454. DOI: https://doi.org/10.1007/s00442-0070932-7

Shavier, G. 1981. Mineral nutrient and nonstructural carbon utilization. In Miller PC ed. Resource use by chaparral and matorral: a comparison of vegetation function in two mediterranean type ecosystems. New York, USA. Springer-Verlag. p. 237-258. 